\title{
Pratiques et représentations dans la conception des intérieurs chez Matali Crasset et Zaha Hadid
}

\author{
Fathia Bouchareb Bellagha
}

\section{OpenEdition}

1 Journals

Édition électronique

URL : http://journals.openedition.org/communicationorganisation/4734

DOI : 10.4000/communicationorganisation.4734

ISBN : : 979-10-300-0155-6

ISSN : 1775-3546

Éditeur

Presses universitaires de Bordeaux

Édition imprimée

Date de publication : 1 décembre 2014

Pagination : 71-84

ISBN : 978-2-86781-905-6

ISSN : $1168-5549$

\section{Référence électronique}

Fathia Bouchareb Bellagha, «Pratiques et représentations dans la conception des intérieurs chez

Matali Crasset et Zaha Hadid », Communication et organisation [En ligne], 46 | 2014, mis en ligne le 01 décembre 2017, consulté le 26 avril 2019. URL : http://journals.openedition.org/

communicationorganisation/4734; DOI : 10.4000/communicationorganisation.4734 


\title{
Pratiques et représentations dans la conception des intérieurs chez Matali Crasset et Zaha Hadid
}

\author{
Fathia Bouchareb ép. Bellagha'
}

\section{Introduction}

Le mot " projet ", par le sens actuellement accepté, renvoie à une distanciation entre le temps de la conception d'un objet et le temps de sa réalisation, une " anticipation opératoire " (Boutinet, $2008: 14$ ).

Alors qu'en architecture, cette notion serait le fait d'anticiper un lieu correspondant à une dimension actancielle à travers une grille de valeurs culturelles (symbolique, fonctionnelle, esthétique, etc.), dans le domaine du design, l'objectif de séduction s'est imposé clairement dès le départ comme opérateur essentiel dans l'intentionnalité de production d'objet. Dans ce cas, le projet prend le sens d'une recherche de qualités formelles tout en performant une fonctionnalité ; ici la communication visuelle est prise en compte au premier chef.

Cette démarche a eu son impact sur les autres domaines du design. Elle sous-tend l'idée de l' "immédiateté " d'une réponse à une demande, ce qui suggère un objectif «explicite » le différenciant du projet qui engage le futur.

L'environnement numérique avec les potentialités qu'il offre est une nouvelle césure dans l'évolution des outils de conception qui semble accentuer cet aspect d'immédiateté, puisqu'il est moyen de modélisation et de simulation virtuelles, qui permet de juger, de choisir et de modifier, avec un rapport au temps très différent de ce qui était d'usage avant son adoption.

Il est indéniable que ces moyens ont facilité le voyage cognitif dans l'espace de l'existant, ce qui pourrait suggérer leur impact sur l'imagination pour atteindre l'espace du possible. Toutefois, au-delà de son aspect instrumental, l'environnement numérique est une interface qui " donne à voir le monde " (Cavallari, 2013), ce qui interpelle la perception des représentations que nous produisons à travers cet environnement : cette perception dure tout le temps de la conception et a un impact sur le produit final.

1 Fathia Bouchareb ép. Bellagha est doctorante, unité de recherche SEA, École Nationale d'Architecture et d'Urbanisme de Tunis (ENAU), Architecte, Enseignante à l'Institut Supérieur des Beaux Arts de Tunis ; fathia_bouchareb@yahoo.fr 
Cela dit, l'hypothèse sous-tendant cette étude est que ces moyens de représentation et de visualisation ont favorisé l'orientation de la pensée vers un registre privilégié de l'espace, celui du visible. Cependant, la pensée du concepteur doit constamment être régulée afin de faire des choix de finalités conscients. L'observation de l'espace de conception de la designer Matali Crasset et del'architecte Zaha Hadid à partir de leurs productions des chambres d'hôtels, offre de la matière pour essayer d'aborder ces questionnements à la lumière de deux approches, l'architecturologie et la sémiotique.

L'architecturologie se place comme la science dont l'objet est la modélisation des opérations cognitives du concepteur, permettant l'élaboration de l'espace architectural, en spécifiant que cet espace architectural est un espace pensé et en cela, il n'est pas toujours équivalent à l'espace construit (Boudon et al., 2000 : 60). Dans son développement, cette science nous livre plusieurs notions et concepts qu'il est nécessaire d'expliciter au niveau de notre étude, afin de rendre compte de leurs champs d'intérêt et éviter toute ambigüité de signification.

L'approche sémiotique permet une lecture des espaces produits comme un continuum à interpréter, à travers une analyse de leurs structures syntagmatiques et sémantiques, ainsi que des formes paradigmatiques caractérisant les contextes de leurs matérialisations. Ces observations éclairent sur l'intentionnalité des concepteurs qui détermine leur rapport au projet et leur rapport au récepteur du projet. La prise en considération de la réception de l'objet conçu est un facteur déterminant dans la démarche conceptuelle et donc dans l'usage et la perception de l'environnement global de la conception.

\section{Méthodologie}

Afin de rendre compte des objets conçus (et réalisés) et de leurs espaces de conceptions, l'observation s'effectue sur un ensemble de supports graphiques (photos, plans), ainsi que sur différents récits et discours des concepteurs concernant leurs projets. L'objectif recherché est de constituer une idée sur le contexte de la conception pour distinguer ce qui est de l'ordre de la contrainte et ce qui constitue un choix tout en essayant de nous distancier par rapport à d'éventuels essais de valorisation.

L'observation liminaire des différentes configurations des chambres proposées ainsi que les différents récits (site de concepteurs, ouvrages, site internet d'hôtels, etc.), permet de dégager des caractéristiques de l'espace conçu qui renvoient à des opérations de référenciation, de découpage et de dimensionnement pertinent dont découlent des mesures qualitatives et quantitatives, en l'occurrence, des échelles architecturologiques.

Il est évident, par ailleurs, que plusieurs échelles opèrent dans la conception, mais, prises séparément, elles n'ont pas la même pertinence.

La première étape de cette analyse est le repérage des échelles qui agissent à un niveau global et celles qui agissent à un niveau local. Par la suite, il 
y a lieu de déterminer l'échelle globale opérant dans la conception par la détermination conjointe d'un ensemble d'échelles. Ce qui pourra permettre de dégager les orientations des démarches conceptuelles (idée, style, finalités, etc.).

\section{Présentation des spécimens}

Le Hi hôtel et le Silken Puerta America sont deux lieux visibles de l'hôtellerie haut de gamme. Les contextes des commandes qui ont régi leur engendrement sont semblables sur plusieurs points:

- les deux hôtels sont situés dans des lieux urbains ;

- l'époque de leur conception est littéralement la même, entre 2003 et 2005 ;

- c'est l'aménagement d'une structure existante ;

- les deux concepteurs ont notifié avoir eu une large liberté dans leur choix conceptuels.

\section{Hi hôtel à Nice}

Pour cet établissement, la designer Matali Crasset « signe avec HI un projet de design total, du graphisme aux objets, de l'architecture à la programmation du lieu $»^{2}$. Elle annonce neuf concepts pour trente-huit chambres (WhiteWhite, Strates...), une conception dont il ressort différentes configurations d'espaces caractérisées par un usage varié de matériaux, de couleurs et de texture.

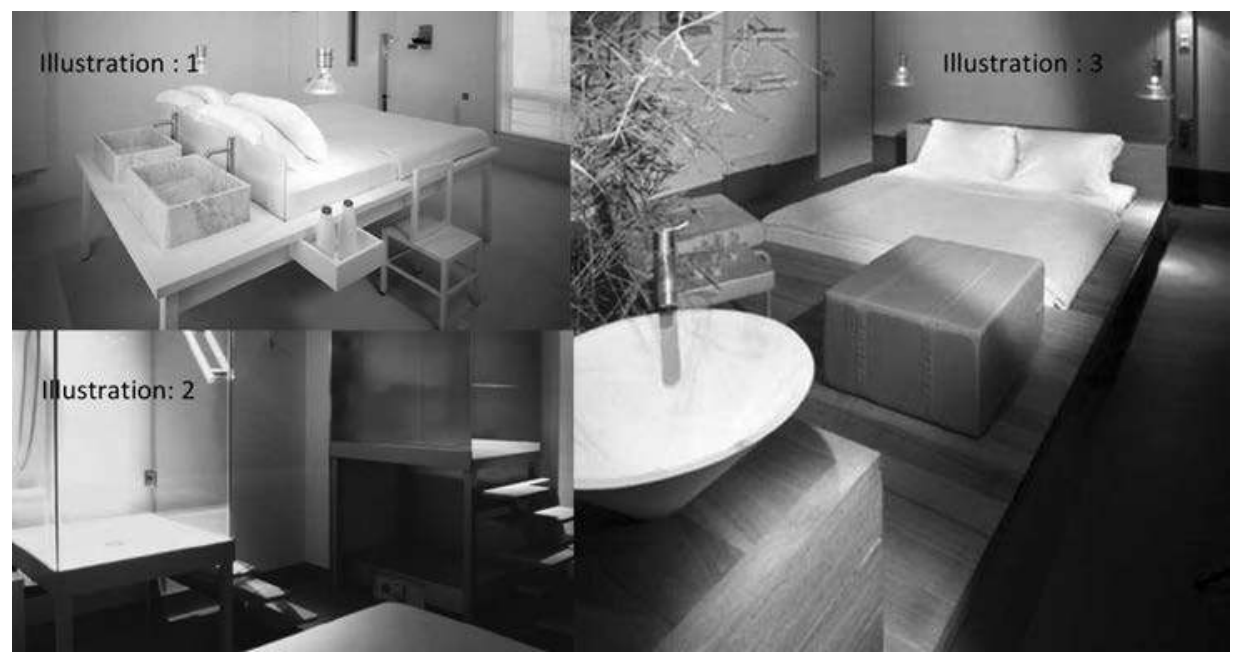

Figure 1 : Chambres du Hi Hôtel.

Illustration 1 : Chambre White White. - Illustration 2 : Chambre Strates Illustration 3 : Chambre Indoor Terrasse (http://www.hi-hotel.net/fr). Photographe : Uwe Spœring

2 Dossier de presse de Hi hôtel. 


\section{Hôtel Silken Puerta America à Madrid}

Zaha Hadid propose trente chambres identiques sur le plan morphologique et organisationnel.

Les couleurs sont homogènes mais varient d'une chambre à l'autre grâce entre autres à l'éclairage. Des changements de teintes peuvent être réglés par l'hôte à l'intérieur de la chambre pour différencier les sous-espaces.

Les espaces sont positionnés selon un cheminement linéaire : hall d'entrée, salle de bain, chambre à coucher avec des séparations murales franches. La chambre semble conçue d'une seule pièce, sans interruption formelle entre le lit et les consoles en guise de tables de chevet, bureaux, etc. Mais aussi, entre les parois et le mobiler, la même logique formelle régit les éléments de la salle de bain.

L'éclairage est une importante composante. Outre la possibilité de varier les couleurs des espaces, l'architecte accompagne la forme du mobilier et des parois impactant la perception pour un effet de flottement de ses différents éléments. Des bandes de LED sont insérées dans les portes pour afficher des messages que l'hôte peut transmettre concernant les services désirés. Un éclairage halogène est encastré aux têtes de lits pour un effet relaxant.

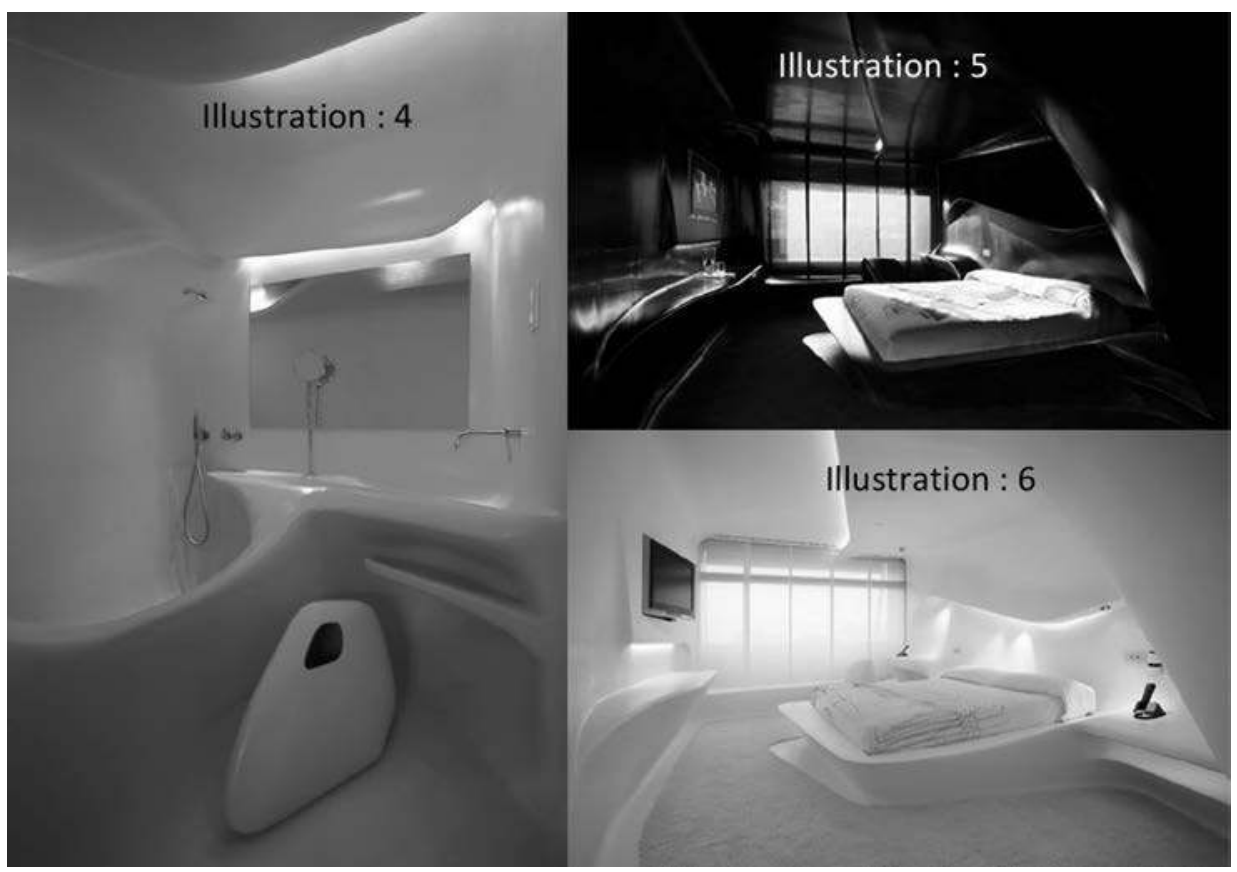

Figure 2 : Hôtel Puerta America

Illustration 4 : Salle de Bain rouge - Illustration 5 : Chambre type noire Illustration 6 : Chambre type blanche (http://www.hoteles-silken.com).

Photographe : Uwe Spœring 


\section{Modélisation de l'espace de conception}

Du point de vue architecturologique, la conception architecturale peut être examinée à la lumière des questions que l'architecte a pu travailler. Ainsi, l'objet conçu et réalisé sera un support d'observation qui permet de distinguer des objets signifiants qui renvoient à une ou plusieurs échelles architecturologiques conjuguées à partir de relations à identifier et à caractériser.

\section{Le repérage des échelles dans l'espace de conception de Matali Crasset}

\section{- L'échelle socioculturelle}

Outre le confort fonctionnel, la conception prend en considération les modes de vie caractérisés par des attentes de liberté et de multiplicité de choix, d'autonomie (travailler, manger ou se relaxer en lieux publics ou privés ; regarder la télé assis, allongé ou en se baignant, commander des boissons ou des plats à tout moment, sans avoir recours au personnel). Grâce à la technologie, ces attentes trouvent une réponse dans la flexibilité et la modularité de l'espace, etc.

- L'échelle de visibilité

Il est patent qu'un choix essentiel est effectué par le designer ; celui d'un décloisonnement de l'espace permettant de regrouper les différentes activités (repos, distraction, bain, etc.), avec un déplacement minime du champ visuel dès l'entrée de la chambre, ce qui crée une visibilité de tous les dispositifs. Le cas emblème est la position de l'espace humide (lavabos, baignoire, etc.), comme partie intégrante de ce champ visuel créant une continuité spatiale, visuelle et kinesthésique entre deux programmes, habituellement, séparés spatialement.

\section{- L'échelle fonctionnelle}

Il est clair que cette échelle est un opérateur global dans la conception : positionner les différents dispositifs suivant des schémas actantiels (Greimas, 1966) qui indiquent des processus, une série d'actions qui varient selon la quête du sujet (le visiteur). L'espace conçu est l'adjuvent catalyseur qui plonge le sujet dans des scénarios, permettant de s'emparer d'expériences distinctes de ce qu'habituellement offre l'hôtellerie de luxe. Cette intention, récurrente du concepteur, est très palpable dans la chambre STRATES où les différentes fonctions sont réparties en niveaux. Le déplacement nécessite un repérage horizontal et vertical, ce qui renouvelle notre rapport aux différents actes du quotidien ainsi que le rapport entre différents sujets partageant l'espace.

- L'échelle de modèle ou référence

Dans le cas de la chambre White-White, l'échelle de modèle de l'espace clinique est manifeste à travers la blancheur totale, la table en guise de lit 
avec une sorte de marchepied avec au-dessus des suspensions pour la lumière. Les produits liquides sont mis dans des bouteilles qui renvoient à celles des produits médicamenteux.

Les chambres INDOOR TERRASSE sont une juxtaposition des lieux d'activités sur un plateau central en bois légèrement soulevé, à la manière des terrasses. La salle de bain limitrophe, est isolée par un rideau végétal. Les couleurs du mobilier sont choisies dans les tons de la nature, avec un vert tilleul pour le textile et un bois au naturel. Les toilettes sont une sorte de cabanon qui prend la forme d'une lanterne, grâce un éclairage intérieur étudié. La disposition des matelas à ras de sol sur le plateau en bois confirme cette intention de renvoyer le visiteur vers une expérience de la terrasse. Dans ce cas, le modèle est un substrat pour configurer l'espace global ; c'est aussi un modèle téléologique pour formuler certains sous-espaces comme la cabane/cabinet.

\section{Le repérage des échelles dans l'espace de conception Zaha Hadid}

\section{- L'échelle géométrique}

Il est clair que l'échelle géométrique se trouve de façon prégnante dans la conception des espaces de Zaha Hadid, fidèle à cette constante position de bannir l'angle droit (Hadid citée in Jodidio, 2012) ; la forme est le résultat d'une "logique fluide de poches interconnectées plutôt que de boîtes " (Silvestri, 2009 : 14). Toutes les formes des artefacts découlent d'une seule peau courbe dénotant une géométrie propre à l'architecture Non-Standard (Silvestri, 2009 : 14).

\section{- L'échelle optique}

Avec des surfaces continues et d'un point de vue optique, il n'existe aucune intersection qui focalise l'œil mais des lignes qui dirigent la vue. Cette continuité est accentuée par des lignes lumineuses qui valorisent l'aspect flottant des éléments composants et l'aspect fluide de la matière. Cela dit, la pertinence optique est présente aussi bien au niveau de la conception des solides qu'au niveau de l'éclairage. Cet élément est très actif dans la perception de l'espace avec les possibilités de variations d'état qu'il offre.

\section{- L'échelle technique}

Les formes libres usitées dans ce projet sont une preuve des multiples possibilités actuelles : nouvelles matières et nouvelles techniques, au niveau du calcul et de la fabrication. La conception de surface sans joint apparent n'est possible qu'avec le recours à des matières et des techniques spécifiques comme c'est le cas du HI-MACS ${ }^{3}$ utilisé dans sept étages du Puerta-Hôtel.

Nous prenons en considération que le travail de conception se fait en équipe et que cette collaboration peut léguer aux autres les opérations de calcul mais si l'on considère que l'échelle technique est constituée par le rapport entre

3 HI-MACS, Matériau composite. Site officiel, disponible sur : http://himacs.fr/ 
une idée et les moyens techniques de sa matérialisation, alors cette échelle devient un élément pertinent essentiel que l'architecte prend nécessairement en compte dans son travail de conception et de mesure dans l'espace.

\section{- L'échelle socioculturelle}

La distinction architecturale et décorative du bâtiment constitue le souci premier des commanditaires d'un hôtel de cette envergure. Cette distinction ne se matérialise pas uniquement au niveau des potentialités de confort offert. Elle est une pertinence qui a régi l'opération de conception de toute la morphologie des éléments de l'espace. Aussi, la cybernétisation des mœurs est prise en compte et a, comme conséquence, l'intégration des possibilités qu'offre la domotique comme opération de pensée qui est en harmonie avec la forme globale qu'offre l'espace : un lieu aux allures futuristes.

\section{- Échelle de modèle}

Comme cela a été déjà évoqué, le schéma organisationnel est un modèle récurent des chambres d'hôtel. Sur le plan morphologique, il est reconnu que ce type de démarche, bien qu'amorcée par des considérations théoriques qui prescrivaient de libérer la forme des logiques normatives et standardisantes de l'architecture moderne, ce choix fournit un registre formel dans lequel l'esthétique de Zaha Hadid trouve à s'inscrire pour en illustrer le modèle.

\section{La relation entre les échelles}

«... On peut faire l'hypothèse que toutes les échelles sont sans doute présentes bien qu'à des degrés divers dans la conception des architectes... » (Boudon, 2005) et c'est essentiellement la relation entre ces échelles qui détermine les orientations conceptuelles. Certes, un repérage exhaustif des échelles architecturologiques serait d'un grand intérêt pour éclairer l'espace de conception. Mais, dans ce travail, nous avons pris délibérément quelques exemples patents qui, à notre avis, se démarquent le plus dans la démarche des deux designers. Par ailleurs, ce qui pourrait démarquer un designer d'un autre c'est aussi la relation entre les échelles!

\section{- Surdétermination}

Nous avons vu dans le cas des chambres de Matali Crasset, que le découpage de l'espace trouve sa pertinence dans l'échelle de visibilité et l'échelle fonctionnelle, un découpage qui a permis de regrouper les éléments de l'espace selon un champ visuel et une scénarisation de l'action. Il est clair que le modèle fonctionnel séparant espace humide et espace sec a servi pour initialiser le découpage de la chambre en deux sous-espaces. Néanmoins, dans la conception de Zaha Hadid, le point de vue dominant est d'ordre optique puisque ce qu'il en ressort est cette attention de saisir de la matière comme une pâte à modeler, d'où surgissent des formes destinées à accueillir des fonctions. 


\section{- Relations en cascade}

D'autres échelles sont initiatrices d'opérations cognitives mais ne sont qu'une conséquence d'autres échelles. Le souci de visibilité a entraîné, chez Matali, un choix de matériaux comme la présence significative du verre ; les considérations socioculturelles ont aussi eu pour conséquences des préoccupations d'ordre technologique avec une domotisation des pièces de vie pour donner plus d'autonomie au visiteur, en l'occurrence par l'automatisation des services tels que les bars en étages. Ici, l'échelle technique est entraînée essentiellement par l'échelle socioculturelle et l'échelle de visibilité. Chez Zaha Hadid, une des suites de la réflexion centrée sur l'aspect formel des objets est la forte pensée technique qui permet le résultat optique escompté.

\section{Les opérations constituant les échelles}

Pour mieux cerner cette idée de l'échelle comme opérateur, nous allons mettre en relief les trois opérations significatives qui le constituent (référenciation, découpage, dimensionnement) tout en insistant sur l'idée de pertinence qui accompagne chaque opération. En rappelant qu' « une telle disjonction n'est pas facile à identifier dans la description d'un processus particulier» (Boudon et al., 2000 : 154) et que l'échelle architecturologique étant l'opération de donner une mesure à un objet en se référant à un domaine, nous essayerons d'identifier ses différentes opérations sans pour autant omettre le caractère construit de leur chronologie.

\section{- La référenciation}

C'est l'orientation de l'espace de référence, le point de vue qui permet de mesurer les objets à découper. Dans sa conception, Matali Crasset se réfère à une variété de domaines pour attribuer des mesures aux objets (espaces et artefacts). Néanmoins, la prééminence de l'échelle de visibilité et de l'échelle fonctionnelle et leur impact dans l'impulsion d'autres échelles en cascade, nous permet de juger qu'elles participent en toute probabilité à la fonction d'initialisation du projet par la fixation d'un point de vue imposé sur la globalité du parti pris.

L'espace de référence étant les classes auxquelles appartiennent les références, ils ont, en majorité, une pertinence socioculturelle des modes de vie comme références susceptibles d'intervenir dans la conception. Et c'est précisément de cette échelle socioculturelle qu'émerge l'échelle de modèle qui emprunte certains objets (tel que le graphique numérique, la cabane, etc.) comme un substrat mis à l'échelle.

Nous rappelons que "la lecture architecturologique d'un édifice découpe l'espace de conception architecturale suivant un ensemble de choix, de décisions dont les pertinences ainsi que les références sont énonçables.» (Boudon, 2005). 
La proposition de Zaha Hadid dénote un engagement du côté de la matière, un travail conceptuel consacré à la forme, la couleur, la lumière prenant sans doute en considération le phénomène de la perception visuelle de l'œuvre. Cela nous permet de stipuler que la pensée est orientée vers les univers visuels et par conséquent vers les moyens d'obtenir les effets escomptés. Dans ce cas d'étude, la référence est morphologique, baignant dans le monde des abstractions géométriques. Rappelons que ces recherches sur la morphologie ne sont pas que représentatives, elles nécessitent un travail sur les possibilités de sa réalisation comme cela a déjà été souligné par Philippe Boudon concernant le cas Bilbao de Frank Gehry. Ce répertoire de forme n'ayant été possible que par les possibilités offertes par le numérique, il en conclut que «le numérique aura bien été espace de référence pour l'architecte, comme, pour prendre un autre exemple, l'économique aura pu l'être pour la maison des artisans chez Le Corbusier, ou comme aujourd'hui le développement durable travaille les esprits. » (Boudon, 2012).

Cela dit, l'hypothèse d'un espace de référence numérique active dans l'espace de conception de Zaha Hadid est tout à fait soutenable.

\section{- Le découpage}

"Le découpage de l'espace de conception se concrétise dans l'espace architectural » (Boudon, 2000 :271). Dans les chambres du HI-Hôtel, l'échelle de visibilité traduit une dimension dans l'échelle de niveau de conception. Elle engage l'ensemble des sous-espaces dans leurs dimensions mais aussi la globalité de l'espace.

Décider de l'entité à mesurer dans le continuum de l'espace de conception relève, ici, d'une intention de créer des unités dynamiques. Les objets n'existent pas isolément mais représentent des syntagmes qui poussent à les exploiter et à se les représenter de différentes façons.

Dans l'hôtel Puerta, le découpage est d'abord spatial, selon une spécialisation des lieux (échelle fonctionnelle). La salle de bain est clairement séparée de l'espace de couchage. Les différents dispositifs sont majoritairement dénotatifs d'une seule fonction.

Aussi et comme nous l'avons déjà spécifié, tout l'espace semble être modelé d'une seule peau lisse et translucide sans discontinuité. Ici, l'image que l'on se fait de la forme de l'espace et donc de comment elle sera perçue est clairement prise en considération. Ce souci patent de donner à l'ensemble une continuité morphologique, par la géométrique, la matière et la texture dénote une scénographie de l'espace selon une pertinence symbolique et géométrique.

\section{- Dimensionnement}

Attribuer des mesures revient à décider de ce qui est à mesurer et de lui attribuer une mesure.

En cela, le dimensionnement est indissociable du découpage et de la 
référenciation. Il est évident que pour des raisons de confort, la mesure initiale se fait très probablement selon l'échelle humaine suivant des considérations ergonomiques. Mais pour les deux cas d'étude, cela ne se fait pas dans l'intention d'une standardisation.

Chez Matali Crasset, la mesure est attribuée selon une pertinence fonctionnelle, incrémentant plusieurs éléments qui, pris séparément, seraient un pur produit industriel, mais c'est leur assemblement qui en fait la dynamique et la singularité de leur dimensionnement.

Pour Zaha Hadid, le support de dimensionnement privilégié est la matière (la peau) qui constitue l'ensemble du solide conçu (on peut supposer que lors de la conception, l'architecte prend en considération les caractéristiques du vide résultant mais ce qui est palpable c'est le travail sur la matière). La pertinence ici est d'ordre formel et optique ce qui engage la pensée vers des considérations techniques rendant la mesure possible.

\section{Conception Versus production}

S'il y a à juger à travers les récits des pourvoyeurs de ces deux lieux ${ }^{4}, l^{\prime} i d e ́ e$ retenue serait que les espaces conçus par les deux concepteurs sont des objets innovants dans le panorama de la production hôtelière.

De quel type d'innovation s'agit-il ? Et quelle est la conséquence de la démarche sur le sens produit ? Une sémiotique pragmatique permet de soumettre l'espace perçu comme un objet à interpréter.

D'une lecture syntagmatique des espaces conçus par Matali Crasset, il en résulte des segments que nous pouvons discriminer sur le plan formel. Aussi, ce sont des objets visuellement dénotatifs, ce qui pourrait suggérer que l'espace est un lieu commun. Néanmoins, si nous raisonnons sur la multiplicité des expériences qu'ils offrent (fonctions rajoutées, nouvelles dispositions, renouvellement de la perception, etc.), cela nous renseigne sur les potentialités engrammées de l'espace de Matali Crasset, peu perceptibles de prime abord mais intelligibles. Il restera à l'expérience d'en rendre compte exclusivement.

Dans les chambres conçues par Zaha Hadid, la fonctionnalité n'est pas déductible d'une segmentation morphologique des objets. Le schéma organisationnel de l'espace est une reprise d'un modèle et c'est la forme de l'ensemble qui est suggestive par des connotations poétiques. Indéniablement, il y a un travail sur l'objet-sculpture (Beyaert-Geslin, 2012 :19) qui se donne à voir, l'espace est customisé sans points de suture comme par un tour de magie.

On pourrait rapprocher cette situation de ce qu'Umberto Eco avait décrit sur le «styling» où il avance : «le "styling" pourrait être (et il est dans la plupart

4 Hôtel Silken Puerta America à Madrid, Site internet de l'hôtel, disponible sur : http://www.hoteles silken. com/content/pdf/pamerica-dossier-de-prensa-en-ingles.pdf ; et le Hi hôtel à Nice, Site internet de l'hôtel, disponible sur : http://www.hi-hotel.net/fr 
des cas) la superposition de nouvelles fonctions secondes à des fonctions premières inchangées » (Eco, 1988 : 298). Architecturalement parlant, sur le plan formel, cela peut correspondre au fait d'habiller une même structure par une variation d'apparats.

Selon lui, cet acte est à but unique. C'est celui de confirmer "par de nouvelles stratégies persuasives, ce que l'usager voulait, faisait, savait faire déjà. Pur acte de persuasion, il ne serait qu'une stratégie adroite d'opinions acquises. » (Eco, 1988 : 298).Et dans l'espace de Zaha l'argument persuasif est d'abord d'ordre formel.

Si nous nous amusons à comparer les deux types d'espaces, celui de Zaha Hadid et celui de Matali Crasset, nous dirons que le premier est évocateur d'images et que le deuxième est un stimulateur d'actions.

\section{Résultats et interprétations}

Si nous confrontons cette lecture de l'espace aux démarches de leur concepteur, ceci n'est pas dans un souci de relier cause à effet ce qui sera de l'ordre du raccourci puisque dans le contexte de notre étude nous ne prétendons pas à l'exhaustivité concernant l'étude des démarches et l'analyse de l'espace conçu. Mais cette mise en relation est faite à dessein de constituer le cadre rendant possible la génération du sens dans un processus de conception.

Nous avons vu que même s'il y a présence des mêmes échelles architecturologiques, cependant, pour les deux concepteurs, ces échelles n'ont pas les mêmes pertinences.

Dans le cas du Hi Hôtel, l'espace de référence relève des classes de situation de vie ; le découpage est fait selon une pertinence socioculturelle. Il est évident que la question qui a été prise en compte c'est la multiplicité des procédures qui implique l'usager ; le dimensionnement porte essentiellement sur le schéma organisationnel qui régit l'ensemble des syntagmes, ce qui nous permet d'identifier un "parti architecturologique» chez Matali Crasset, support de son travail. Il s'agit bien de l'ensemble \{'échelle socioculturelle, échelle fonctionnelle\} qui conjointement donnent des pertinences à des intentions initiales.

Le «parti architecturologique» chez Zaha Hadid est constitué de l'ensemble \{échelle optique, échelle formelle symbolique\} puisque nous avons bien vu que l'espace de référence relève des classes d'univers visuel, le découpage dissocie la fonction de la forme, puisque le schéma fonctionnel est découpé selon une échelle de modèle et la forme est créée selon une pertinence optique engageant par là-même un travail de dimensionnement sur les surfaces.

\section{Conclusion}

Dans cet article, nous avons essayé de rendre compte de la relation entre démarche conceptuelle et produit, entre projet et design. Nous avons eu recours d'une part à l'architecturologie pour approcher les opérations de conceptions 
à partir de l'espace conçu, ce qui a permis de dégager les points de vue qui ont présidé aux choix conceptuels. D'autre part, la sémiologie était un moyen de dégager le sens de ces productions tout en prenant en considération leur ancrage dans le contexte de leur génération.

Nous avons décelé dans le travail de la designer Matali Crasset, une mise en relation de différents dispositifs ; l'espace est un dispositif, ce qui suppose un travail sur des «scénarios de vie», engageant en particulier le plan comme support de représentation des possibilités d'action.

Dans l'espace de chambre conçu par l'architecte Zaha Hadid, nous avons trouvé que le dispositif est figé avec une intention de faire varier son image par l'abstraction géométrique ; l'intérieur n'est plus construit par des murs et un plafond mais entouré d'une peau qui suggère de prendre toute forme tant que la technique et le numérique le permettent. Cette démarche ébranle l'idée des intérieurs comme lieux de «scène de vie» où c'est la pratique qui génère et décore l'espace pour le mettre en scène ; l'espace est exposé pour être vu et être touché sans qu'on le fasse bouger.

Enfin, nous sommes consciente qu'un bon éclairage sur l'espace de conception ne peut se passer de l'exhaustivité tant sur le plan du repérage des échelles architecturologiques et des relations qui les régissent, que sur le plan de la multiplication des cas d'étude. L'exercice, auquel nous nous sommes livrée, est une sorte d'esquisse d'un outil d'investigation que l'élargissement du corpus pourra parfaire dans la perspective d'interroger au mieux, la relation entre projet et design, entre le monde idéal du concepteur et son produit.

\section{BIBLIOGRAPHIE}

BEYAERT-GESLIN Anne (2012), Sémiotique du design, PUF (Formes sémiotiques). BOUDON Philippe (1991), "Le point de vue de la mesure dans la conception architecturale : de la question d'échelle à l'échelle comme question ». In Philippe Boudon (dir.), De l'Architecture à l'Épistémologie, la question de l'échelle, Paris, PUF, p. 169-195. (Nouvelle Encyclopédie Diderot)

BOUDON Philippe et al. (2000), Enseigner la conception architecturale, Cours d'architecturologie, Paris, Éditions de la Villette.

BOUDON Philippe (2002), "La notion d'échelle et les catégories de Ch. S. Peirce». In Anne d'HENAULT (dir.), Questions de sémiotique, nouv. Éd. Paris, PUF, p. 467-480.

Philippe BOUDON (2005), « De l'espace architectural à l'espace de conception. In PROJETAR 2005. II Seminário sobre Ensino e Pesquisa em Projeto de Arquitetura [page consultée le 28 mai 2014]. Disponible sur : http://projedata.grupoprojetar.ufrn.br/ dspace/bitstream/123456789/1190/1/236\%20BOUDON_P.pdf

BOUDON Philippe (2012), Nul n'entre ici s'il (n') est géomètre », DNArchi, 4 avril. Disponible sur : http://dnarchi.fr/pedagogie/nul-nentre-ici-sil-nest-geometre/

BOUTINET Jean-Pierre (1990), Anthropologie du projet, Paris, PUF, Paris, réed. 2008. 
BOUTINET Jean-Pierre (1993), Psychologie des conduites à projet, Paris, PUF (Que sais-je ? $\mathrm{n}^{\circ}$ 2770).

CAVALLARI Peppe (2013), "Une réflexion philosophique inédite sur le web, Lecture de «L'être et l'écran. Comment le numérique change la perception » de Stéphane Vial (Puf 2013), Sens public, 19 décembre. Disponible sur : http://sens-public.org/spip. php?article1051\&lang=fr

ECO Umberto (1988), Le signe, Paris, Le Livre de poche.

ECO Umberto (1999), Kant et l'ornithorynque, Paris, Grasset.

GREIMAS Algirdas Julien (1966), Sémantique structurale : recherche et méthode, Paris, Larousse.

HENAULT Anne, dir. (2002), Questions de sémiotique, Paris, PUF.

JODIDIO Philip (2012), Zaha Hadid, Paris, Taschen. (Petite collection)

LATOUR Bruno (2005), Nous n'avons jamais été modernes : Essai d'anthropologie symétrique, Paris, La Découverte.

LEBAHAR Jean-Charles (2007), La conception en design industriel et en architecture : désir, pertinence, coopération et cognition, Paris, Lavoisier.

MIDAL Alexandra (2012), Matali Crasset, Paris, Norma.

MIDAL Alexandra (2013), Design, l'Anthologie, St Étienne, Cité du Design.

ROSSI Jean-Gérard, (2000), « Notes sur la sémiotique de Peirce ». In Processus du sens. Sociologues en ville, Numéro 2. Paris, L'Harmattan, p. 213-220.

SILVESTRI (2009), Perception et conception en architecture non-standard. Une approche expérimentale pour l'étude des processus de conception spatiale des formes complexes, thèse de Doctorat en Mécanique Génie Civil, Université Montpellier II Sciences et Techniques du Languedoc, Juin.

Résumé : Nous nous proposons d'étudier l'espace de conception en prenant comme exemple la démarche d'une designer et celle d'une architecte à travers leurs productions des espaces de chambres d'hôtels, qui sont respectivement Matali Crasset et Zaha Hadid. Une designer qu'on reconnaît, orientée vers le projet, et une architecte reconnue par son empreinte à travers ses objets. L'étude se base sur le travail de rapprochement effectué par Philippe Boudon entre architecturologie et sémiotique. Elle fait appel à l'architecturologie pour comprendre les mécanismes cognitifs de la conception à partir de l'espace conçu, en l'occurrence une matérialisation de ses opérations et en tant que tel, c'est un ensemble de signes indiciels du travail du concepteur. Ainsi, l'utilisation de l'approche sémiotique permettra d'interpréter le sens de la production et de décrire les relations entre les démarches conceptuelles et leurs résultats.

Mots-clés : projet, design, architecturologie, sémiologie.

Abstract: We propose to study the design space using the example of tow designer's approaches: that of a designer and an architect through their productions spaces of hotel rooms. They are respectively Matali Crasset a designer that recognizes oriented project and Zaha Hadid an architect recognized by her footprint through her architectural objects. The study is based on 
work done by Philippe Boudon rapprochement between architecturology and semiotics. We use architecturology to understand the cognitive mechanisms of the designed space's form, which in this case is a materialization of its operations and as such, it is a set of index signs of the designer's labor. Thus, using the semiotic approach, we will interpret the meaning of the production and describe the relationships between conceptual approaches and their results.

Keywords: project design, architecturology, semiotics. 